C2006 IEEE. Personal use of this material is permitted. However, permission to reprint/republish this material for advertising or promotional purposes or for creating new collective works for resale or redistribution to servers or lists, or to reuse any copyrighted component of this work in other works must be obtained from the IEEE. 


\title{
ASPECTS INFLUENCING TRUSTWORTHINESS IN SERVICE ORIENTED ENVIRONMENTS
}

\author{
Farookh Khadeer Hussain ${ }^{1}$, Elizabeth Chang ${ }^{1}$ and Tharam S. Dillon ${ }^{2}$ \\ ${ }^{1}$ School of Information Systems, Curtin University of Technology, Perth, Australia \\ \{Farookh.Hussain,Elizabeth.Chang\}@cbs.curtin.edu.au \\ ${ }^{2}$ Faculty of IT, University of Technology, Sydney, Australia \\ tharam@it.uts.edu.au
}

\begin{abstract}
Transactions have moved away from faceto-face encounters to more being on the Internet. The infrastructure for the business and information exchange activities could be client-server, peer-to-peer (P2P), or mobile networks. In this paper we refer to 'peer' as an entity involved in a transaction. Trust between two interacting peers involved in an electronic transaction is a major issue that needs to be addressed in order to make the internet a safe medium for carrying out transactions. In this paper, we propose the aspects that can influence the trustworthiness assigned to the trusted peer. Furthermore we catalogue these factors into three classes and discuss the relationship between these factors.
\end{abstract}

Keywords: Trust, Factors, Trusting Peer, Trusted Peer

\section{Introduction}

The advent of the Web and its intrusion into business, commerce, government and the health sector have led to Web based ecommerce for business interactions and collaborations over great distances and at any time. In the last ten years, this new Web based environment has enabled economic growth, industry development, technology innovation, and resource sharing.[12] This new business environment has led to the development of the Service-oriented environment, that transcends the previous static, closed, competitive models and has moved towards flexible, open, collaborative, sharing and distributed environments that are able to respond in a timely manner to consumer needs and business dynamics inherent in the networked economy[12].

However, these have also introduced challenges. One of the most pressing of these arises from the fact that in a business or social interaction on the Internet, we cannot rely on the usual physical, facial and verbal cues that we might have relied on to reach a judgement as to whether or not the other party will fulfill the service which they are promising. In addition, in the case of the purchase of physical goods over the Internet, we have no direct physical, sensory contact with the specific product and are reliant solely on the promise of the seller[12]. These factors and several others, when taken together, create the imperative for being able to make judgments within such an environment about the other parties' trustworthiness and capability to provide the service at a specific level of quality. Through adopting new trust technology in the Service-oriented network environment, a platform for both consumers and businesses to learn from each other is created. Thus, real business value, increased consumer confidence, guaranteed quality of product and service could become a reality in the virtual word [12].

In this paper we study the factors which determine the trust that the trusting agent has in the trusted agent. This paper is organized as follows

Section 2- Reviews the existing work

Section 3, Section 4, Section 5 and Section 6 present the factors that determine the trust which the trusting agent has in the trusted agent.

Section 7 concludes the paper.

\section{Existing Work}

Egger [1, 4, 7, 8, 9], proposed set of factors which, if given due importance while designing an interface of a website (or HumanComputer Interaction), can induce trust in the human users of the websites. These factors can communicate trust to the human users $[1,4$, 7, 8, 9]. Egger[1, 4, 7, 8, 9] takes a bigger view and considers how factors like the usability of the website, the way content is organized, how security and privacy issues are addressed by the website, can communicate trust to the human users of these websites. The factors proposed by Egger are applicable for B2C e-commerce, where the consumer 
(usually the client) interacts with the service providers through websites. We feel that the factors proposed by him are applicable primarily to $\mathrm{B} 2 \mathrm{C}$ e-commerce and not to service oriented environments in which the infrastructure for the business and information exchange activities could be client-server, peer-to-peer (P2P), or mobile networks.

Kim and Moon [5] investigated which graphic design elements in a website can communicate trust to the human users. They, however, do not investigate how the content and the usability of the website can assist in communicating trust to the users. Moreover, they have a much narrower scope than Egger because they claimed that these trust-inducing features were applicable to only the Korean population.

Our domain and motivation is totally different from the above mentioned approaches, which focus on $\mathrm{B} 2 \mathrm{C}$ communications and the factors, if given due importance while making websites, that communicate trust to the human users. We focus on service oriented environments and we examine the factors which influence the trusting peer in deciding whether to trust the trusted peer. Additionally, we examine the psychological factors that influence the trusting peer. For further discussion throughout this paper we make use of the terms trusted peer [3] and trusting peer [3]. The trusting peer has to make a decision whether to trust the trusted peer for a given interaction.

\section{Aspects Affecting Trust}

In this section, we present the factors that can communicate trust to the trusting peer in peer-to-peer e-commerce. We catalogued these factors into three classes, namely:

1. Pre-interaction Factors

2. Reputation Factors

3. Personal Interaction Factors

These catalogues, in turn, consist of other factors. In the following subsections, we define and provide examples of the factors in each catalogue.

\section{Pre-interaction Factors}

We define Pre-interaction Factors as define Pre-interaction Factors as 'those factors which can influence the trusting agent, whether or not to trust the trusted agent, before any interaction between the trusting agent and the trusted agent takes place'. We identify the following three factors in this catalogue:

i. Psychological nature of the trusting peer ii. Attitude of the trusting peer towards ecommerce

iii. Previous interactions with the trusted peer

We now explain each of these factors and provide examples.

\section{i. (Catalogue 1) - Psychological Nature of the Trusting Peer}

We believe that the psychological nature of the trusting peer is a very important factor that influences decisions as to whether they should trust the 'trusted' peer or not. Persons with 'Sensing' preference have a tendency to rely on facts and experience $[10,11]$. On the contrary, persons with 'Intuition' preference have a tendency to rely more on possibilities and taking risks $[10,11]$. We believe that people with sensing preference will not trust any person with whom they did not have any previous interaction. Conversely, we believe that people with intuition preference may trust a person with whom they have not had any previous interactions. Depending on whether the trusting peer has sensing or intuition preference, this preference will influence it's decision to trust a given trusted peer with or without detailed information collection of the trustworthiness of the trusted peer.

Persons with 'Thinking' preference have a tendency to analyze things in an objective and logical fashion with little or no regard for personal values, before they reach or take a decision [10, 11]. Persons with 'Feeling' preference place primary importance on personal values, before reaching a decision [10, 11]. We believe that if the trusting peer has a thinking preference, he/she will pay little or no importance to personal values of the trusted peer, personal feelings with the trusted peer and make an objective and logical decision whether to trust the trusted peer or not. On the other hand, trusting peers who give preference to feeling will place greater importance on his/her personal feelings of the trusted peer and values of the trusted peer while they decide whether to trust the trusted peer.

Depending on the psychological type of the trusting peer, whether he/she gives preference to 'thinking' or 'feeling', will determine whether he/she make the decision through facts or through the personal values of the trusted peer.

\section{ii. (Catalogue 1) - Attitude or Mindset of the Trusting Peer towards Peer-to-Peer E-Commerce}

This is another important factor which will have an influence on the trusting peer, in deciding whether it should or should not trust the trusted peer. As we mentioned previously, 
with the advent of the internet and its subsequent ubiquitous use, all business transactions were carried out over the internet. However, many people were reluctant to use this medium as a means of carrying out transactions due to the inherent risks involved in electronic business. Many people regarded it as unsafe as they were not totally convinced about how the other entity behaves in things like possessing credit card details, handling privacy issues....

Although technologies like cryptography, digital certificates and various legislation rules have been introduced to mitigate the risk of carrying online transactions, some sections of the populace are still not convinced that the internet is a safe place to carry out transactions, if certain defensive measures are followed. This is the general attitude of the entity towards electronic commerce. An example of such a defensive measure is the verification of the identity of the website with the help of digital certificates before carrying out an electronic transaction.

In peer-to-peer communication, the problem is graver as compared to client-server communication. In client-server communication much of the security measures taken to ensure that the client-server based ecommerce is a safe place to carry out transactions rely on Trusted Certification Authorities. In P2P communication, on the other hand, there can be no central authority due to its decentralized nature. Hence, much of the security measures used in client-server communication that can induce trust in consumers cannot be used in P2P communication.

A lot depends on the attitude and mindset of the trusting peer towards the e-commerce. Peer-to-peer e-commerce has far less security guarantees and far more risks involved compared to client-server based e-commerce.

\section{iii. (Catalogue 1) - Previous Interactions}

The outcome of previous transactions between the trusting peer and the trusted peer will have a major bearing on the decision whether to trust the trusted peer again. Depending on the outcome of the previous transaction, the trusting peer will be more confident in deciding whether to trust the trusted peer or not. If the outcomes of the previous transactions are positive then its trust in the trusted peer will grow, and the trusting peer is most likely to trust the trusted peer in future transactions. On the contrary, if the outcome of the previous transaction was negative, this will have a negative impact on the perceived trustworthiness of the trusted peer by the trusting peer.

The above mentioned three factors, namely the psychological nature of the trusting peer, attitude of the trusting peer towards ecommerce and previous interactions have an affect on the trusting peer in determining whether to trust another peer or not, before any interaction with the trusted peer take place. Hence, we have collectively named these factors as Pre-interaction Factors.

\section{Reputation Factors}

A trusting agent in order to make a trust based decision of whether or not to interact with a given trusted agent asks other agents about the trustworthiness of the trusted agent. These other agents communicate an indication of the trustworthiness of the trusted agent. This method of asking the other agents in the network about the trustworthiness of the trusted agent can help the trusting agent in deciding whether it should trust the trusted agent. We call this gathered information on the trustworthiness of the trusted agent its 'Reputation'. We define reputation factors 'as those factors pertaining to the reputation of the trusted agent and can influence the trusting agent in deciding whether to trust the other agent or not'. We identify four major factors pertaining to the reputation of the trusted agent, which can influence the decision of the trusting agent:

$\begin{aligned} \text { iv. } & \text { Trusted Reputation } \\ \text { v. } & \text { Unknown Reputation } \\ \text { vi. } & \text { Positive Reputation } \\ \text { vii. } & \text { Negative Reputation }\end{aligned}$

\section{iv. (Catalogue 2) - Trusted Reputation, and \\ v. (Catalogue 2) - Unknown \\ Reputation}

As mentioned previously, in order to find the trustworthiness' of a peer, the trusting peer asks other accessible peers about the trustworthiness of the trusted peer. Any peer present in the network can respond to the trusting peer's request for information regarding the trustworthiness of the trusted peer. Malicious peers may reply with a deceptive trust value; increased or decreased trust value for the trusted peer. Malicious peers may respond with a trust value even if the trusted peer has not had an interaction with them.

In order to counter this problem, we propose that the trusting peer, classify the 
reputation that it acquires from contemporary peers in the network into three broad groups namely:

- $\quad$ Reputation obtained from peers who it trusts to give accurate recommendations. For discussion purposes we term them trusted or trustworthy peers.

- $\quad$ Reputation obtained from peers who it does not trust to give accurate recommendations. We term them as untrusted or untrustworthy peers.

- Reputation obtained from peers with whom it does not have an experience of soliciting reputations. We term them unknown peers.

The trusting peer, over a period of time, can come to know which peers report truthful and accurate trustworthiness values and which peers give misleading, deceitful trust values. We propose that Reputation obtained from trustworthy peers be called Trustworthy Reputation and that obtained from untrustworthy peers be called Untrustworthy Reputation. Additionally, the trusting peer may receive trustworthiness' values from other peers with whom it has no previous experience of soliciting recommendations and hence their recommendations cannot be classified as either trustworthy reputation or untrustworthy reputation. We propose that such a reputation be known as an Unknown Reputation. Unlike the untrustworthy reputation which is fraudulent, unknown reputation can be fraudulent or truthful.

We propose that when the trusting peer receives the reputation of the trusted peer from other peers in the network, it should disregard the untrustworthy reputation. It should put more credence on trustworthy reputation and some weight on the unknown reputation (as it is not sure whether this reputation is trusted or not). We believe that the two main factors that aid the trusting peer in deciding whether it should trust the trusted peer or, in other words, the factors which induce trust in the trusting peer are trustworthy reputation and unknown reputation, since the trusting peer, takes these two into account before deciding whether to trust the trusted peer or not. It disregards the un-trusted reputation as fraudulent.

\section{vi. (Catalogue 2) - Positive Reputation, \\ and \\ vii. (Catalogue 2) - Negative \\ Reputation}

The trustworthy peers and the unknown peers can give a positive or negative recommendation about the trusted peer. These reputation values of the trusted peer communicated by the trustworthy peers and the untrustworthy peers can influence the trusting peer to a great extent, in deciding whether it should trust the trusted peer or not.

If the trusting peer receives a significant positive reputation from trustworthy peers and new (unknown) peers, it can have a great affect while deciding to trust the trusted peer. On the contrary, if it received significant negative reputation from the trustworthy peers and unknown peers, this can communicate to the trusting peer not to trust the trusted peer. Hence, both positive and negative reputation of the trusted peer obtained from the trustworthy and unknown peers can aid the trusting peer in deciding whether it should trust the trusted peer. Since the trusting peer disregards the reputation of the trusted peer, obtained from un-trustworthy peers, the reputation obtained from untrustworthy sources is an insignificant factor when deciding whether to trust.

\section{Personal Interaction Factors}

We define Personal Interaction Factors 'as those factors which help the trusting agent to associate a trustworthiness value to the trusted agent based on its personal interaction with the trusted agent'. Based on these personal interaction factors, the trusting agent can assigning a specific trust value to the trusted agent and decide whether to trust the trusted agent in the future.

We identify two main factors in this catalogue. They are:

viii. Negotiated Behavior or Mutually Agreed Behavior

ix. Correlation

\section{viii. (Catalogue 3) - Negotiated Behavior or Mutually Agreed Behavior}

We define the expected behaviour of the trusted agent (from the perspective of the trusting agent) as 'the mutually agreed conduct of the trusted agent prior to its interaction with the trusting agent'.

The trusting agent, before carrying out a transaction with the trusted agent, enters goes through the negotiation phase. In the negotiation phase, the trusting agent lays down the expected behaviour of the trusted agent. The expected behaviour can be regarded as a collection of the set of activities that the trusting agent expects the trusting agent to perform. The trusted agent then considers whether or not it can deliver on the expected behaviour. If the trusted agent thinks that it can 
deliver on the expected behaviour, then the expected behaviour becomes 'Mutually Agreed Behaviour'. I term it as Mutually Agreed Behaviour because the trusting agent will determine the trustworthiness value of the trusted agent in the interaction, by comparing the actual behaviour of the trusted agent in the interaction with the mutually agreed behaviour.

On the contrary if the trusted agent feels that it cannot deliver on the expected behaviour, then the trusted agent will modify the expected behaviour by deleting those activities in the expected behaviour which it cannot deliver on or by adding some activates which it feels could help the trusting agent achieve its objectives in the transaction. The trusted agent would then communicate the expected behaviour, modified by it to the trusting agent. The trusting agent would then consider whether the modified expected behaviour by the trusted agent would achieve the objectives that it is looking for in the interaction. If the trusting agent feels that the modified expected behaviour would help it achieve its objectives in the interaction then the modified expected behaviour then it would communicate to the trusted agent it's willingness to interact with the trusted agent based on the modified expected behaviour. The modified expected behaviour then becomes the Mutually Expected Behaviour.

On the contrary if the trusting agent feels that its objectives in the transaction cannot be met based on the modified expected behaviour by the trusted agent then it may choose not to carry out the transaction with the trusted agent. The trusting agent may choose to propose a new version of the modified expected behaviour of the trusted agent from the perspective of the trusting agent. The negotiation process can then be carried on the till both the trusting agent and the trusted agent agree upon the mutually agreed behaviour.

\section{ix. (Catalogue 3) - Correlation}

We define correlation as 'the degree of parallelism between the mutually agreed demeanour of the trusted agent and actual demeanour of the trusted agent during interaction'. Correlation refers to the degree of correspondence between the following two factors:

- The Mutually Agreed Behaviour of the trusted agent

- The Actual Behaviour of the trusted agent.

The greater the correlation between the 'mutually agreed behaviour' and the 'actual behaviour', the higher will be the trustworthiness value assigned to the trusted agent by the trusting agent and vice versa. Strong correlation between the above mentioned factors indicates that the trusted agent delivered mostly on the mutually agreed behaviour and hence the trustworthiness value assigned by the trusting agent to the trusted agent would be high. On the other hand, a weak correlation between the mutually agreed behaviour and the expected behaviour indicates that the trusted agent failed to deliver on the mutually agreed behaviour, and hence the trusting agent would assign a low trustworthiness value to the trusted agent.

\section{Conclusion}

Through detailed studies, we proposed nine factors of trust in E-Commerce and catalogued them into three classes. We found that the existing literature does not present the components of trust that influence trust. Our future work involves showing how trust is built, maintained and destroyed using these factors.

\section{References}

[1] Bhiru Shelat, Florian N.Egger, F. 2002, What Makes People Trust Online Gambling Sites?,Available: [http://www.ecommuse.com/research/publicati ons/chi2002.pdf] (10/08/2003).

[2] BJ Fogg, Hsiang Tsueng., The Elements of Computer Credibility

Available:[http://delivery.acm.org/10.1145/310 000/303001/p80-

fogg.pdf?key $1=303001 \&$ key $2=8303186701 \& \mathrm{c}$ oll $=$ portal $\& \mathrm{dl}=\mathrm{ACM} \& \mathrm{CFID}=16753875 \& \mathrm{CFT}$ OKEN=3600767 ](10/10/2003).

[3] Farookh Khadeer Hussain, Elizabeth Chang, Tharam Dillon 2004, 'State of the Art in Trust in Ad-Hoc Peer-to-Peer (P2P) Networks', in International Conference of Computer Applications, Myanmar.

[4] Florian N.Egger, Boyd de Groot. 2000, Developing a Model of Trust for Electronic Commerce: An Application to a Permissive Marketing Web Site, Available: [http://www.ecommuse.com/research/publicati ons/WWW9.htm] (20/6/2003).

[5] Jinwoo Kim, Jae Yun Moon, Emotional Usability of Customer Interfaces, Available:

[http://hci.yonsei.ac.kr/non/e02/97-CHI-

Emotional_Usability_of_Customer_Interface.p df]

$(23 / 08 / 2003)$. 
[6] Myers, S. 2003, Working out your Myers Briggs type, Available: [http://www.teamtechnology.co.uk/tt/tarticl/mb-simpl.htm] (27/12/2003).

[7] N.Egger, F., "Trust Me, I'm an Online Vendor":Towards a Model of Trust for ECommere System Design, Available: [http://www.zurich.ibm.com/ mrs/chi2000/con tributions/egger.html] (10/09/2003).

[8] N.Egger, F., Towards a Model of Trust for E-Commerce System Design, Available:

[http://www.zurich.ibm.com/ mrs/chi2000/con tributions/egger.html] (29/05/2003).

[9] N.Egger, F. 2003, Deceptive Technologies:Cash, Ethics \&HCI, Available: [http://www.ecommuse.com/research/publicati ons/sigchi_bulletin.htm] (23/05/2003).

[10] Yao-Hua Tan, Walter Thoen. 2000, The Formal Aspects of a Generic Model of Trust for Electronic Commerce, Available: [http://www.computer.org/proceedings/hicss/0 493/04936/04936006.pdf] (2003).

[11] G.Mallach, E. 2000, Decision and Data warehouse Systems, Irwin Mc Graw Hill Companies.

[12] Chang, E., Dillon, T.S., Hussain, F.K., 'Trust and Reputation for Service Oriented Environment', John Wiley and Sons(To be published in 2005).

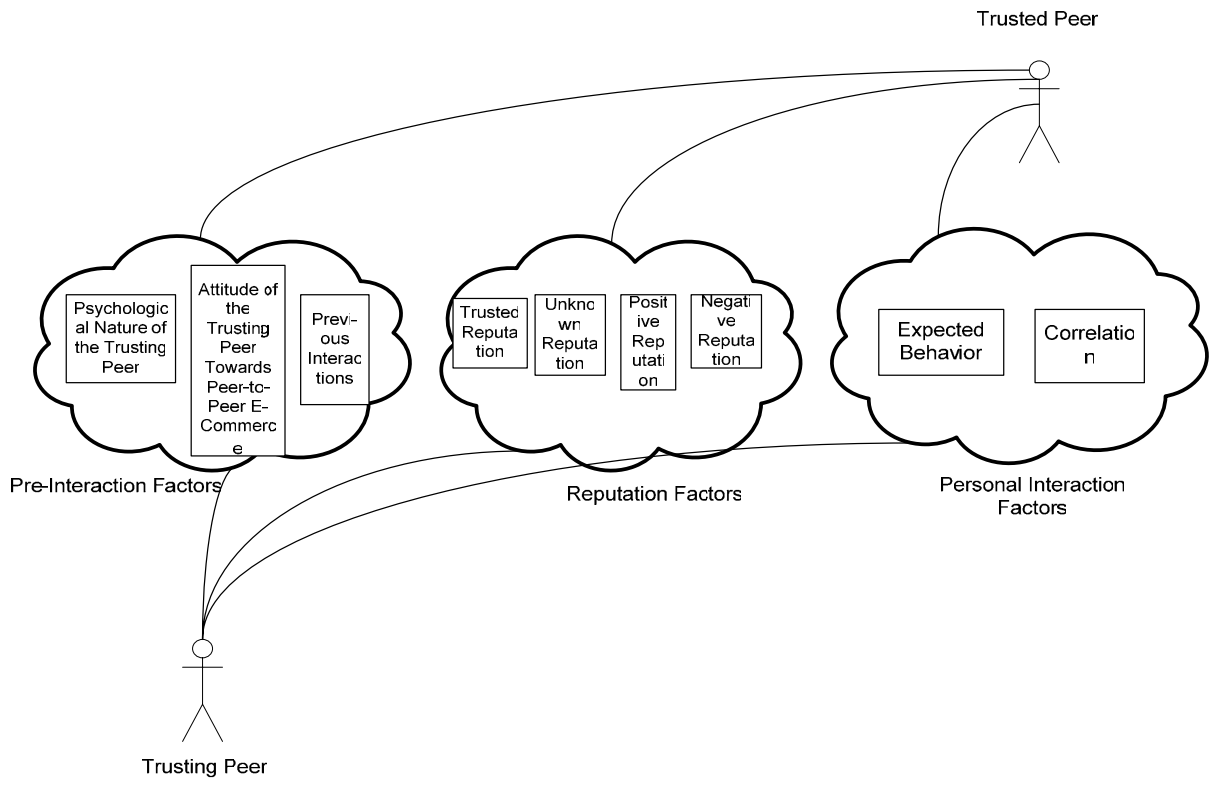

Figure1: Overview of the Factors in Service Oriented Environments

Pre-Interaction Factors

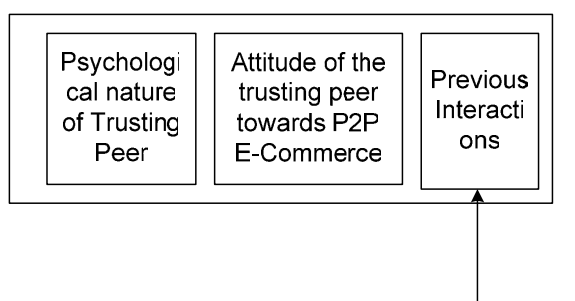

Reputation Factors

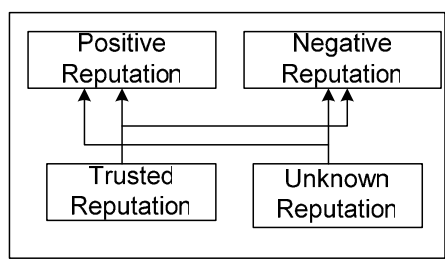

Personal Interaction Factors

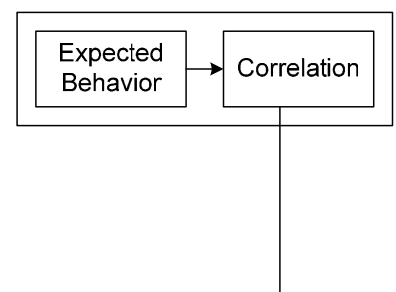

Figure 2: Relationship between the Individual Catalogues and Individual Factors 